\title{
Second-look breast ultrasonography after galactography in patients with nipple discharge
}

\author{
Hyeonseon Kim', Bong Joo Kang², Sung Hun Kim², Jeongmin Lee² \\ ${ }^{1}$ Department of Radiology, Uijeongbu St. Mary's Hospital, ${ }^{2}$ Department of Radiology, Seoul St. Mary's Hospital, \\ College of Medicine, The Catholic University of Korea, Seoul, Korea
}

\begin{abstract}
Aim: The objective of this study was to evaluate the diagnostic performance of second-look breast ultrasound (US) immediately after galactography in patients with nipple discharge. Materials and methods: Between January 2010 and November 2018, 89 patients with nipple discharge were retrospectively analyzed. All patients were examined by galactography and US. US was performed twice, before and after galactography. Galactography, mammography and US findings were categorized according to the Breast Imaging Reporting and Data System (BI-RADS). The final diagnosis was established by histopathological examination. Results: Out of 89 patients, 25 (28.1\%) patients had positive findings as evident by US before galactography. Forty-eight (53.9\%) patients had positive findings as demonstrated by galactography. Fifty-nine $(66.3 \%)$ patients had positive findings as evident by second-look US after galactography (13 patients with benign, 37 with borderline and 9 with malignant lesions). Second-look US after galactography showed the highest diagnostic accuracy (83.2\%), sensitivity (95.8\%) and negative predictive value $(93.3 \%)$ for borderline and malignant lesions. For the detection of malignant lesions, secondlook US after galactography showed the highest sensitivity (90\%) and negative predictive value (96.7\%). Agreement between imaging and histological result using Bland-Altman analysis exhibited no significant difference between the size established by ultrasound and galactography. Conclusion: The second-look US after galactography is a powerful diagnostic tool for the detection of lesions in patients with nipple discharge and demonstrates high diagnostic accuracy, sensitivity and negative predictive value.
\end{abstract}

Keywords: nipple discharge; breast cancer; ultrasound; galactography

\section{Introduction}

Nipple discharge is a common complaints and cause anxiety and mental discomfort as it may be associated with breast cancer. Even though the majority of cases

Received 08.07.2019 Accepted 15.11.2019

Med Ultrason

2020, Vol. 22, No 1, 58-64

Corresponding author: Professor Bong Joo Kang, MD, PhD

Department of Radiology,

Seoul St. Mary's Hospital, College of

Medicine, The Catholic University of Korea

222, Banpo-daero, Seocho-gu,

Seoul, 06591, Republic of Korea

Phone: 82-2-2258-6253

Fax: 82-2-599-6771

E-mail: gmlionmain@gmail.com have benign etiologies, in about $10-15 \%$ of patients' discharge is due to cancer or precancerous mastopathy [1]. Therefore, it is important to differentiate the physiologic discharge during breastfeeding or medication-related conditions from pathologic causes. If the discharge is bilateral and milky or serous, the condition is not considered as bothersome and significant. However, the color or laterality of discharge cannot be solely used as a tool to rule out the presence of an underlying malignancy.

The diagnostics of nipple discharge is challenge to clinicians. The diagnostic algorithm for pathologic nipple discharge includes clinical breast examination, mammography and whole breast US as the first line of investigations [2-4]. If the outcome is negative, galactography as a second-line procedure should be recommended [5]. 
Other imaging modalities, such as contrast-enhanced breast magnetic resonance imaging (MRI), are recommended only for selected patients, in cases of failure of galactography or in the preoperative settings when other techniques are inconclusive. Second-look US could be used as an additional breast imaging method to correlate the lesion identified from galactography.

Galactography was considered for many years to be the key imaging modality for assessing nipple discharge, being useful in identify the location of intraductal lesions [6]. Recently, the role of galactography was questioned [7], especially related to the use of high-resolution US. However, galactography is cost-effective, readily available, and easy to perform and interpret. For example, without preoperative mapping with galactography, breast surgeons would underestimate the disease and fail to perform accurate central duct excision [8,9].

To the best of our knowledge, no previous research has investigated the efficacy of combining galactography and the high-resolution US to detect abnormalities in patients with nipple discharge. We hypothesized that the application of second-look US after galactography would generate higher diagnostic accuracy. For this reason, the aim of our study was to evaluate its diagnostic performance and usefulness in daily practice.

\section{Materials and Methods}

\section{Study population}

We retrospectively reviewed 104 patients with nipple discharge evaluated in the radiology department of our hospital between January 2010 and November 2018 by galactography and US (pre- and in the 5-31 days postgalactography), and underwent either percutaneous USguided biopsy or excisional biopsy. Patients lost during follow-up ( $\mathrm{n}=2)$, those without biopsy $(\mathrm{n}=2)$, with duplicate use of same imaging study $(n=4)$ and those without second-look US after galactography $(\mathrm{n}=7)$ were excluded. Consequently, 89 patients were included for statistical analysis.

\section{Data collection}

Medical records were reviewed and the following parameters were included in our database for analysis: age, clinical symptoms, personal history, type of nipple discharge, laterality, menopausal status and histopathologic results. Archived images of mammography, US, and galactogram scans were interpreted by a radiologist with over 15 years of experience in breast radiology, blinded to pathologic outcomes. Depending on age, the patients were divided into two groups: under and over 50 years. Based on the aspect of the nipple discharge, this was classified also into two groups: serous or bloody.
The mammography and US findings were categorized using the American College of Radiology Breast Imaging Reporting and Data System (BI-RADS) [10]. Also, if a mass was found on mammography or US, the maximal diameter of the mass was recorded and then categorized into 3 groups according to its pathologic findings; benign, borderline and malignant. Atypical ductal hyperplasia and intraductal papillomas were classified into borderline group.

\section{Galactography technique}

One of two breast radiologists with over 15 years of experience performed galactography using digital 2D full-field mammography (Hologic Lorad Selenia; Danbury, USA) and evaluated the galactographic images. While the patient was in a sitting position, the nipple orifice, which was secreting discharge, was located using a magnifying lens. A heat lamp was applied to the nipple for 10 minutes to relax the periareolar sphincter muscle. The nipple and periareolar area were sterilized with an alcohol swab followed by a povidone swab. Gentle periareolar pressure was applied to elicit discharge and a 27-gauge blunted needle attached to a syringe filled with $1 \mathrm{~cm}^{3}$ iopromide (Ultravist 370; Bayer Schering Pharma, Seoul, Korea) was inserted into the discharging pore. After completion of infusing the contrast media (approximately $0.3-1.0 \mathrm{~cm}^{3}$ ), the patients received mammography or magnification mammography in a craniocaudal or mediolateral view. Recannulation, reinjection and additional mammography were carried out if necessary $[11,12]$. The Galactogram Image Classification system (GICS) was used [13] during the process of interpreting the archived galactography images.

\section{Second-look US}

Although the term 'second-look' is used even when there is no initial US examination, we only included patients who went through US before galactography to compare the diagnostic power of US alone to combined galactography and second-look US. Second-look US was performed by one of two breast radiologists with over 15 years of clinical experience and paid special attention to the detected lesion from galactography. US was performed using the IU22 system (Philips Medical Systems, Bothell, WA, USA), which was equipped with a $50-\mathrm{mm}$ array transducer with a bandwidth of 5-12 MHz.

\section{Statistical analysis}

Statistical analysis was performed using the SAS system for Windows version 9.2 (SAS Institute Inc., Cary, NC, USA). Chi-square test and Fisher's exact test were used to assess the associations between the parameters such as age, symptoms, personal history, menopausal status, laterality, size, and imaging modalities with statistical significance set at $p<0.05$. We ran two types of 
statistical analysis. The first outcome represents 'benign vs. borderline and malignancy' and the second outcome show 'benign and borderline vs. malignancy'.

McNemar's test was used to determine the relationship between the chosen imaging modalities and the histopathological results. Sensitivity, specificity, overall accuracy, and positive (PPV) and negative predictive (NPV) values were calculated. We used univariate logistic regression to calculate the odds ratio and $95 \%$ confidence interval. Finally, by using Bland-Altman analysis, we compared the two methods of measurement with scatter plots (US vs. pathology and Galactography vs. pathology) where the difference between the measured sizes against their means was represented. The mean difference between the imaging modalities (US, Galactography) and the histological results was calculated and then related to $95 \%$ confidence interval of mean difference and limits of agreement. Size variation on imaging vs. pathology was reported as a median and interquartile range using Box plots [14].

\section{Results}

The patients' demographic data are detailed in Table I. For the pathologic results, a benign lesion was observed in 41 out of $89(46.1 \%)$ patients (35 fibrocystic change, 2 fibroadenoma, 2 stromal fibrosis and one ductal hyperplasia/ductectasia), borderline pathology in 38 (42.7\%) patients (36 intraductal papilloma and 2 atypical ductal hyperplasia) and malignant lesion in $10(11.2 \%)$ patients ( 8 ductal carcinoma in situ and 2 invasive ductal carci- noma). As a result, benign and borderline lesions were more common than malignant lesions for patients with a nipple discharge. Furthermore, a significant difference in the size between benign and borderline lesions compared to malignant lesions $(\mathrm{p}=0.0139)$ was found.

Out of 89 patients, 25 (28.1\%) patients were identified with positive findings by US before galactography, ( 4 benign, 14 borderline and 7 malignant lesions). Forty-eight $(53.9 \%)$ patients were identified with positive findings using galactography (10 interpreted as benign lesions, 30 as borderline and 8 as malignant). During second-look US after galactography, 59 (66.3\%) patients were identified with positive findings (13 patients with benign lesions, 37 with borderline lesions and 9 with malignant lesions). The associations between nipple discharge characteristics, imaging techniques findings and histopathological results are presented in Table II.

Table III shows the diagnostic performance of image modalities for detecting 'borderline and malignant lesions' and malignant lesion only. Employment of second-look US after galactography showed the highest diagnostic accuracy $(83.2 \%)$, sensitivity $(95.8 \%)$, and NPV (93.3\%) for the detection of borderline and malignant lesions. However, PPV (78\% vs. $84 \%$ ) and specificity (68.3\% vs. $90.2 \%)$ was lower compared to the initial US. Diagnostic accuracy of US before galactography was $65.2 \%$ and that of galactography was $77.5 \%$. For the detection of malignant lesions, second-look US after galactography exhibited the highest sensitivity $(90 \%)$ and NPV (96.7\%) (fig 1).

Table I. Patients' characteristics

\begin{tabular}{|c|c|c|c|c|}
\hline & Total & Benign & Borderline & Malignant \\
\hline \multicolumn{5}{|l|}{ Age, $n(\%)$, years } \\
\hline$<50$ & $53(59.5)$ & $21(39.6)$ & $27(50.9)$ & $5(9.4)$ \\
\hline$\geq 50$ & $36(40.4)$ & $20(55.5)$ & $11(30.5)$ & $5(13.8)$ \\
\hline \multicolumn{5}{|l|}{ Discharge, n (\%) } \\
\hline Serous and others & $28(31.4)$ & $17(60.7)$ & $11(39.2)$ & 0 \\
\hline Bloody & $61(68.5)$ & $24(39.3)$ & $27(44.2)$ & $10(16.3)$ \\
\hline \multicolumn{5}{|l|}{ Personal Hx*, n (\%) } \\
\hline No & $72(80.9)$ & $29(40.2)$ & $33(45.8)$ & $10(13.8)$ \\
\hline Yes & $17(19.1)$ & $12(70.5)$ & $5(29.4)$ & 0 \\
\hline \multicolumn{5}{|c|}{ Menopausal status, n (\%) } \\
\hline Pre & $78(76.4)$ & $32(47.0)$ & $30(44.1)$ & $6(8.8)$ \\
\hline Post & $21(23.6)$ & $9(42.8)$ & $8(38.0)$ & $4(19.0)$ \\
\hline \multicolumn{5}{|l|}{ Laterality, n (\%) } \\
\hline Bilateral & $5(5.6)$ & $4(80.0)$ & $1(20.0)$ & 0 \\
\hline Unilateral & $84(94.3)$ & $37(44.0)$ & $37(44.1)$ & $10(11.9)$ \\
\hline Size (mm) & 51 & 6 & 35 & 10 \\
\hline Mean (SD) & $10.49(13.0)$ & $5.33(2.5)$ & $10.49(4.4)$ & $27(22.1)$ \\
\hline
\end{tabular}


Table II. Association between radiological and pathological results

\begin{tabular}{|c|c|c|c|c|c|c|c|}
\hline & Total & Benign & $\begin{array}{l}\text { Borderline + } \\
\text { Malignant }\end{array}$ & p-value & $\begin{array}{l}\text { Benign + } \\
\text { Borderline }\end{array}$ & Malignant & p-value \\
\hline Discharge & & & & 0.028 & & & $<0.0001$ \\
\hline Serious and others & $28(31.5)$ & $17(60.7)$ & $11(39.2)$ & & $28(100.0)$ & 0 & \\
\hline Bloody & $61(68.5)$ & $24(39.3)$ & $37(60.6)$ & & $51(83.6)$ & $10(16.3)$ & \\
\hline MMG & & & & $<0.0001$ & & & 0.3173 \\
\hline Negative & $75(92.6)$ & $37(49.3)$ & $38(50.7)$ & & $69(92.0)$ & $6(8.0)$ & \\
\hline Positive & $6(7.4)$ & 0 & $6(100.0)$ & & $3(50.0)$ & $3(50.0)$ & \\
\hline Initial US & & & & $<0.0001$ & & & 0.0011 \\
\hline Negative & $64(71.9)$ & $37(57.8)$ & $27(42.2)$ & & $61(95.3)$ & $3(4.6)$ & \\
\hline Positive & $25(28.1)$ & $4(16.0)$ & $21(84.0)$ & & $18(72.0)$ & $7(28.0)$ & \\
\hline Galactography & & & & 1 & & & $<0.0001$ \\
\hline Negative & $41(46.1)$ & $31(75.6)$ & $10(24.3)$ & & $39(95.1)$ & $2(4.8)$ & \\
\hline Positive & $48(53.9)$ & $10(20.8)$ & $38(79.2)$ & & $40(83.3)$ & $8(16.6)$ & \\
\hline Second-look US & & & & 0.0045 & & & $<0.0001$ \\
\hline Negative & $30(33.7)$ & $28(93.3)$ & $2(6.7)$ & & $29(96.6)$ & $1(3.3)$ & \\
\hline Positive & $59(66.2)$ & $13(22.0)$ & $46(77.9)$ & & $50(84.7)$ & $9(15.2)$ & \\
\hline
\end{tabular}

The results are expressed as number (\%). MMG: mammography, US: ultrasonography.

Table III. The performance of mammography, US, galactography, and second-look US in identifying malignant lesions

\begin{tabular}{|c|c|c|c|c|c|c|c|c|c|c|}
\hline & \multicolumn{5}{|c|}{ Borderline + Malignant } & \multicolumn{5}{|c|}{ Malignant } \\
\hline & $\operatorname{Acc}(\%)$ & $\mathrm{Se}(\%)$ & $\mathrm{Sp}(\%)$ & PPV (\%) & NPV (\%) & $\operatorname{Acc}(\%)$ & $\operatorname{Se}(\%)$ & $\mathrm{Sp}(\%)$ & PPV (\%) & NPV (\%) \\
\hline Discharge & 60.7 & 77.1 & 41.5 & 60.7 & 60.7 & 42.7 & 100.0 & 35.4 & 16.3 & 100.0 \\
\hline MMG & 53.1 & 13.6 & 100.0 & 100.0 & 49.3 & 88.8 & $33.3 \mathrm{w}$ & 95.8 & 50.0 & 92.0 \\
\hline Initial US & 65.2 & 43.8 & 90.2 & 84.0 & 57.8 & 76.4 & 70.0 & 77.2 & 28.0 & 95.3 \\
\hline Galactography & 77.5 & 79.2 & 75.6 & 79.2 & 75.6 & 52.8 & 80.0 & 49.3 & 16.6 & 95.1 \\
\hline Second-look US* & 83.2 & 95.8 & 68.3 & 77.9 & 93.3 & 42.7 & 90.0 & 36.7 & 15.2 & 96.6 \\
\hline
\end{tabular}

MMG: mammography, US: ultrasonography, PPV: positive predictive value, NPV: negative predictive value; Acc: accuracy; Se: sensitivity; Sp: specificity.

* The result of second-look US after galactography = the result of combined second-look US and galactography

Agreement between the imaging and the histological results showed no significant differences between both groups (sonographic size versus galactographic size) according to Bland-Altman analysis. There was no significant underestimation of size using US. When galactography was used to measure the size of the lesion, it reflected relatively well the actual tumor size based on the histological data (fig 2).

\section{Discussion}

Nipple discharge is a common symptom observed in routine clinical practice and in most of the cases is related to a benign condition. However, if the condition is spontaneous and shows bloody content, careful analysis becomes imperative to exclude any malignant diseases. It is important not only to identify the presence of disease but also to discriminate the causes of the discharge [15].

Borderline lesions, such as intraductal papilloma, are well-known to be the most common cause of nipple discharge; hence their detection is of utmost importance. Galactography has traditionally been the method of choice for the evaluation of patients with a nipple discharge. The method directly shows the secreting duct, which is cannulated and opacified through the contrast medium injection. However, it does not always display specific findings, so it could be difficult to build a differential diagnosis between benign and malignant ductal lesions [16]. Moreover, it could be difficult to cannulate the duct, especially in patients with intermittent discharge or nipple retraction $[16,17]$. Another important issue is related to the use of ionizing radiation that could be harmful in young patients [16].

With the development of modern US equipment with high-frequency transducers, breast US has been increasingly used as an alternative method [17]. In several studies, the efficiency of US has been demonstrated in the evaluation of distal lesions in obstructed ducts as compared with galactography $[10,16,18]$. US is also advantageous in the assessing the relationship of lesion with the 


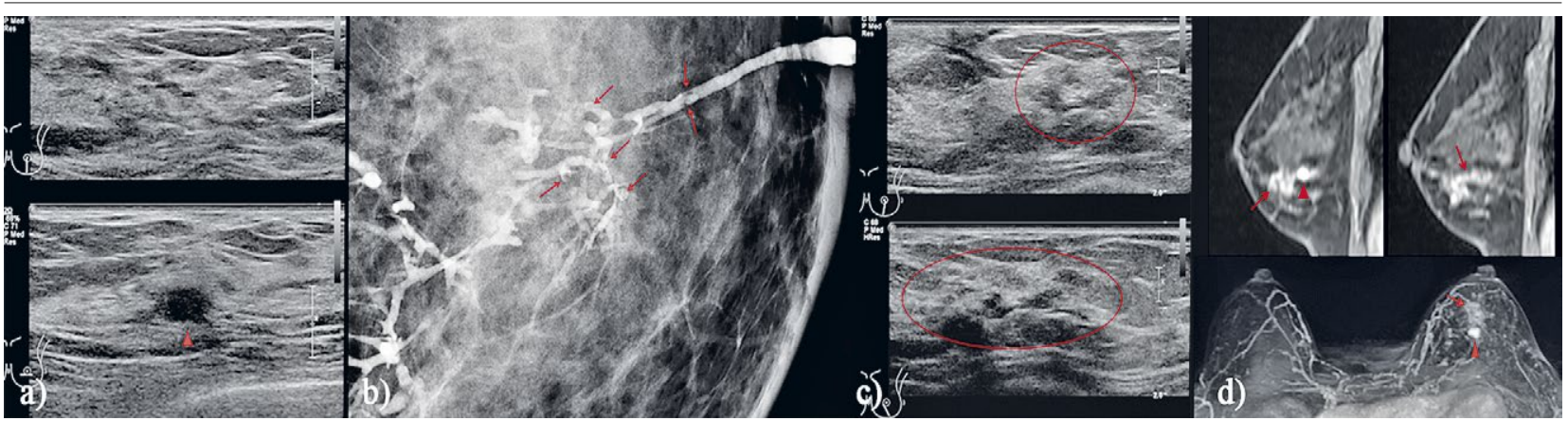

Fig 1. A 44-year-old premenopausal woman with bloody nipple discharge: a) Initial US showing non-specific clustered cystic lesion at 6 o'clock direction (BI-RADS 2, benign finding) and another small angulated marginated hypoechoic mass far away from nipple were detected at 6 o'clock direction of Lt. breast (BI-RADS 4A, low suspicion for malignancy) (arrowhead); b) Conventional galactography on craniocaudal direction shows multiple irregular filling defects, suggesting suspicious lesions (arrows); c) Second-look US was performed immediately after galactography shows segmental duct dilatations in the corresponding area which is the left central breast (BI-RADS 2, benign finding $\rightarrow$ 4B, moderate suspicion for malignancy) (circles). Again, the small hypoechoic mass was found at 6 o'clock direction (4A); d) Axial and sagittal early dynamic contrast-enhanced MR images show segmental non-mass enhancement (arrows) in the left central breast that can be correlated to the lesion found on the second-look US. The homogenous enhancing lesion (arrowheads) in the 6'o clock direction seen in MR imaging correlates with a different lesion seen far away from the nipple from second-look US. The final surgical pathologic result of non-mass enhancement (arrows) on MR imaging was DCIS and the small enhancing lesion (arrowheads) was mucinous carcinoma.

involved ductal system, the characteristics of the lesions, and the uncovering of periductal lesions. However, US has limited values in discovering microcalcifications and for the lesions not associated with ductal dilatation [16]. A recent study revealed the diagnostic performance, the sensitivity, specificity, PPV and NPV, of breast US in the detection of lesions in patients with pathological nipple discharge as $75 \%, 42.9 \%, 11.1 \%$, and $94.7 \%$ and that of galactography as $100 \%, 38.1 \%, 13.3 \%$, and $100 \%$, respectively [10]. We found that second-look US after galactography showed high sensitivity for 'borderline and malignant lesions' (95.8\%) and malignant lesions only $(90 \%)$.

Magnetic resonance imaging (MRI) is known as a highly sensitive technique for invasive breast cancer (68-100\%) and DCIS (77-96\%) detection [19,20]. Several studies have been conducted to evaluate the potential diagnostic role of MRI in ductal pathologies and have provided a comparative analysis between MRI and galactography $[14,21,22]$. A study reported a statistically significant difference in overall sensitivity between the two methods. Particularly, MRI showed higher sensitivity (97.78\%) compared with galactography $(48.89 \%)$ for the identification of ductal pathologies. However, for both methods, the specificity was $100 \%$ [14]. Even if galactography represents the standard imaging technique for evaluating patients with nipple discharge, it is not always available and the likelihood to differentiate between benign and malignant lesions could be controversial. A few studies suggest that nipple discharge is a valid indication to perform MRI [14]. However, the role of breast MRI in
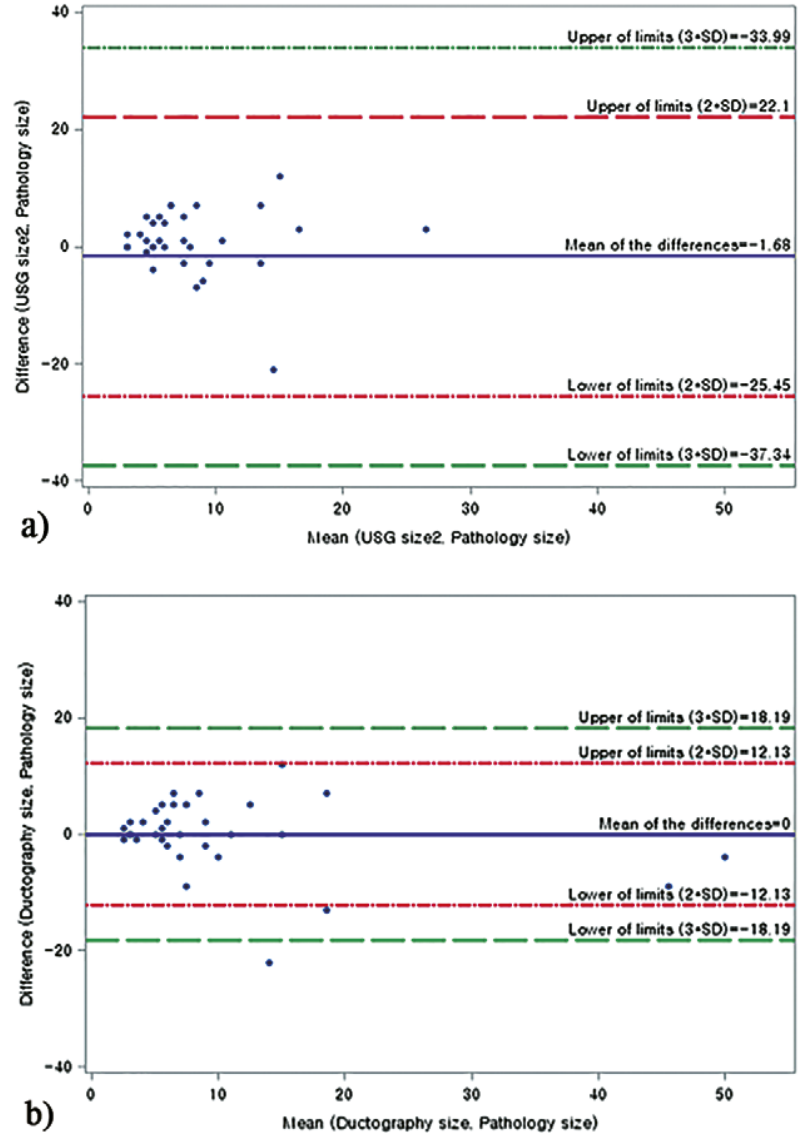

Fig 2. Bland-Altman plots illustrate the size difference between galactography (a), US (b) and the histological tumor size. Compared to US, the size measured from galactography is more likely to correspond well with the size of the histological result. 
evaluating nipple discharge is still controversial [22-24]. Current indication for breast MRI, according to EUSOBI guidelines, does not include the evaluation of patients presenting with nipple discharge [23].

Our research revealed that combined galactography and second-look US led to great diagnostic performance for detecting any kind of lesions in patients with nipple discharge. It also revealed that the lesions identified using US and galactography are mostly benign or borderline lesions. Istomin et al, reported the probability of malignancy in patients with pathologic nipple discharge as low as $2.7 \%$ [5]. This finding is concordant with our research.

Based on our prior knowledge about the importance of identifying borderline lesions, we were able to describe two different outcomes: benign lesion vs. "borderline and malignant lesions', and 'benign and borderline lesions' $v s$. malignant lesions. Second-look US after galactography exhibited the highest diagnostic accuracy $(83.2 \%)$, sensitivity (95.8\%), and NPV (93.3\%) for detection of borderline and malignancy lesions. It also showed high sensitivity (90\%) and NPV (96.7\%) for the identification of malignant lesions. However, low accuracy (42.7\%), specificity $(36.7 \%)$ and PPV (15.3\%) were observed for identifying malignant lesions. These results demonstrate that second-look US after galactography is a reliable tool for the identification of the source of nipple discharge (regardless of the nature of discharge) including borderline and malignant lesions.

As various high-tech imaging modalities have become available, the use of galactography as a second-line diagnostic investigation has been a matter of debate over many studies [26]. Dinkel et al pointed out that galactographic finding of benign and malignant lesions overlap with no specific signs and does not aid in the prediction of true histology of these lesions [27]. Although this problem is associated with many diagnostic imaging modalities, we could postulate, based on our research, that second-look US would help in anticipating the nature of the lesions. In addition, it would help the radiologists to reevaluate the unnoticed lesions from the initial US. Furthermore, as demonstrated in the Bland-Altman plot, galactography is advantageous because it agrees well with the size of the histological result compared to US.

As we mentioned earlier, Istomin et al [5] suggested galactography as a second line procedure if other modalities' result came out negative. Our research is in concordance with the conclusion of their study. The difference in our research is that we emphasized the importance of second-look US in addition to galactography.

This study has several limitations. First of all, it is retrospective in design. Yet, our study included only consecutive patients in the data analysis. In addition, we routinely perform second-look US immediately after galactography in our clinical practice. Subsequently, it was feasible to conduct this retrospective analysis. Galactography and second-look US were performed and interpreted by radiologists specialized in breast radiology with immense experience. Nonetheless, US could show interobserver variability. Finally, there is no defined protocol for second-look US. Detailed indications and guidelines for second-look US are further required for a more precise evaluation [12].

\section{Conclusion}

In patients with nipple discharge, second-look US after galactography should be considered as a powerful diagnostic tool for the detection of lesions: our research exhibited its high diagnostic accuracy, sensitivity, and NPV.

\section{Conflict of interest: none}

Acknowledgment: The statistical consultation was supported by the Department of Biostatistics of the Catholic Research Coordinating Center, Catholic Medical Center.

\section{References}

1. Zylstra S. Office management of benign breast disease. Clin Obstetrics Gynecol 1999;42:234-248.

2. Moriarty AT, Schwartz MR, Laucirica R, et al. Cytology of spontaneous nipple discharge - is it worth it? Performance of nipple discharge preparations in the College of American Pathologists Interlaboratory Comparison Program in Nongynecologic Cytopathology. Arch Pathol Lab Med 2013;137:1039-1042.

3. Rissanen T, Reinikainen H, Apaja-Sarkkinen M. Breast sonography in localizing the cause of nipple discharge: comparison with galactography in 52 patients. J Ultrasound Med 2007;26:1031-1039.

4. Sanders LM, Daigle M. The rightful role of MRI after negative conventional imaging in the management of bloody nipple discharge. Breast J 2016;22:209-212.

5. Istomin A, Masarwah A, Pitkanen M, et al. Galactography is not an obsolete investigation in the evaluation of pathological nipple discharge. PLoS One 2018;13:e0204326.

6. Tabár L, Dean PB, Péntek Z. Galactography: the diagnostic procedure of choice for nipple discharge. Radiology 1983;149:31-38.

7. Berger N, Luparia A, Di Leo G, et al. Diagnostic Performance of MRI Versus Galactography in Women With Pathologic Nipple Discharge: A Systematic Review and MetaAnalysis. AJR Am J Roentgenol 2017;209:465-471.

8. Cardenosa G, Doudna C, Eklund GW. Ductography of the breast: technique and findings. AJR Am J Roentgenol 1994;162:1081-1087. 
9. Woodward S, Daly CP, Patterson SK, Joe AI, Helvie MA. Ensuring excision of intraductal lesions: marker placement at time of ductography. Acad Radiol. 2010;17:1444-1448.

10. D’Orsi CJ, Sickles EA, Mendelson EB, Morris EA, et al. ACR BI-RADS ${ }^{\circledR}$ Atlas, 5th edition. Breast Imaging Reporting and Data System. Reston, VA, American College of Radiology; 2013.

11. Kim SH, Cha ES, Kim HS, Kang BJ, Choi JJ, Jung JH. Galactography acquired with digital mammography in patients with nipple discharge: a retrospective analysis. Arch Gynecol Obstet 2009;280:217-222.

12. Jung HK, Park YM, Baek HJ, et al. Comparison Between Ultrasonography and Galactography in Detecting Lesions in Patients With Pathologic Nipple Discharge. Ultrasound Q 2019;35:93-98.

13. Berna-Serna JD, Torres-Ales C, Berna-Mestre JD, SolaPerez J, Canteras-Jordana M. Galactography: an application of the Galactogram Imaging Classification System (GICS). Acta Radiol 2010;51:128-136.

14. Hong MJ, Cha JH, Kim HH, et al. Second-look ultrasonography for MRI-detected suspicious breast lesions in patients with breast cancer. Ultrasonography 2015;34:125-132.

15. Gruber IV, Rueckert M, Kagan KO, et al. Measurement of tumour size with mammography, sonography and magnetic resonance imaging as compared to histological tumour size in primary breast cancer. BMC Cancer 2013;13:328.

16. Slawson SH, Johnson BA. Ductography: how to and what if? Radiographics 2001;21:133-150.

17. Manganaro L, D’Ambrosio I, Gigli S, et al. Breast MRI in patients with unilateral bloody and serous-bloody nipple discharge: a comparison with galactography. Biomed Res Int 2015;2015:806368.

18. Ballesio L, Maggi C, Savelli S. Adjunctive diagnostic value of ultrasonography evaluation in patients with sus- pected ductal breast disease. Radiol Med 2007;112:354365.

19. Hild F, Duda VF, Albert U, Schulz KD. Ductal orientated sonography improves the diagnosis of pathological nipple discharge of the female breast compared with galactography. Eur J Cancer Prev 1998;7:S57S62.

20. de Paula IB, Campos AM. Breast imaging in patients with nipple discharge. Radiol Bras 2017;50:383-388.

21. Chen L, Zhou WB, Zhao Y, et al. Bloody nipple discharge is a predictor of breast cancer risk: a meta-analysis. Breast Cancer Res Treat 2012;132:9-14.

22. Orel SG, Schnall MD. MR imaging of the breast for the detection, diagnosis, and staging of breast cancer. Radiology 2001;220:13-30.

23. Morrogh M, Morris EA, Liberman L, Borgen PI, King TA. The predictive value of ductography and magnetic resonance imaging in the management of nipple discharge. Ann Surg Oncol 2007;14:3369-3377.

24. Wenkel E, Janka R, Uder M, et al. Does direct MR galactography have the potential to become an alternative diagnostic tool in patients with pathological nipple discharge? Clin Imaging 2011;35:85-93.

25. Mann RM, Kuhl CK, Kinkel K, Boetes C. Breast MRI: guidelines from the European society of breast imaging. Eur Radiol 2008;18:1307-1318.

26. Lorenzon M, Zuiani C, Linda A, Londero V, Girometti R, Bazzocchi M. Magnetic resonance imaging in patients with nipple discharge: should we recommend it? Eur Radiol 2011;21:899-907.

27. Dinkel HP, Trusen A, Gassel AM, et al. Predictive value of galactographic patterns for benign and malignant neoplasms of the breast in patients with nipple discharge. Br J Radiol 2000;73:706-714. 